\title{
Study of Percolative Transitions with First-Order Characteristics in the Context of CMR Manganites
}

\author{
J. Burgy and E. Dagotto \\ National High Magnetic Field Lab and Department of Physics, Florida State University, Tallahassee, FL 32306 \\ M. Mayr \\ Max Planck Institute für Festkörperforschung, Heisenbergstraße 1, 70569 Stuttgart, Deutschland
}

(Dated: October 28, 2018)

\begin{abstract}
The unusual magneto-transport properties of manganites are widely believed to be caused by mixed-phase tendencies and concomitant percolative processes. However, dramatic deviations from "standard" percolation have been unveiled experimentally. Here, a semi-phenomenological description of Mn oxides is proposed based on coexisting clusters with smooth surfaces, as suggested by Monte Carlo simulations of realistic models for manganites, also briefly discussed here. The present approach produces fairly abrupt percolative transitions and even first-order discontinuities, in agreement with experiments. These transitions may describe the percolation that occurs after magnetic fields align the randomly oriented ferromagnetic clusters believed to exist above the Curie temperature in Mn oxides. In this respect, part of the manganite phenomenology could belong to a new class of percolative processes triggered by phase competition and correlations.
\end{abstract}

PACS numbers: 71.10.-w, 75.10.-b, 75.30.Kz

\section{INTRODUCTION}

The understanding of the self-organized behavior of transition-metal oxides, such as manganites and cuprates, has developed into one of the dominant scientific themes of condensed matter physics. Phase coexistence and competition between different kinds of orders involving charge, orbital, lattice, and spin degrees of freedom leads to physical "complexity" as a characteristic of their behavior. The balance between competing phases is subtle, and small changes in composition, magnetic fields, or temperatures lead to large changes in the material properties. In the context of colossal magnetoresistance (CMR), these phenomena motivated the nanoscale phase separation theory of manganited, and its concomitant percolative transition from the metal to the insulator. Many experiments have reported results compatible with phase separation and percolation in $\mathrm{Mn}$ oxidest细. Recently, CMR has been observed in simulations of simple models of competing phases, between $T_{\mathrm{C}}$, the ordering temperature, and $T^{*}>T_{\mathrm{C}}$, the temperature where coexisting clusters start developing upon cooling In the presence of magnetic fields the preformed ferromagnetic (FM) clusters align their magnetic moments, and they percolate. This encouraging agreement between theory and experiments suggests that the basic concept behind the CMR phenomenon -phase competition- has been unveiled.

However, several experimental facts still await for a rationalization within the phase separation scenario. Notorious among them is the abrupt character of the metalinsulator transition (MIT) in some manganites, result incompatible with standard percolative phenomena where the transition is smooth. The results in Fig.1 illustrate this paradox: (a) In $\mathrm{La}_{5 / 8-y} \operatorname{Pr}_{y} \mathrm{Ca}_{3 / 8} \mathrm{MnO}_{3}$, the residual resistivity $\rho_{d c}$ at zero temperature $T$ changes fast
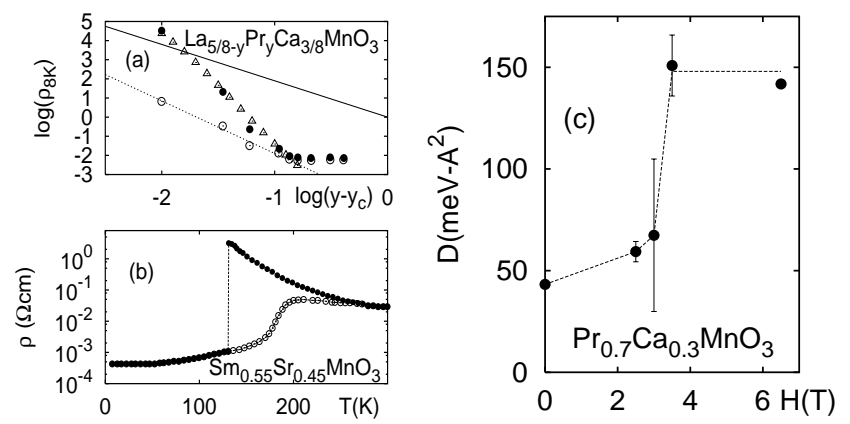

FIG. 1: Results illustrating the "abrupt" character of the metal-insulator transition in some manganites (compositions indicated). (a) Dependence of regidual $\rho_{d c}$ on chemical substitution at $0(-)$ and $4 \mathrm{kOe}(\mathrm{O}) \mathrm{l}$. The solid line represents the prediction of standard percolation, while the dotted one is a guide to the eye. The $\triangle$ are results of a simulation using a broad distribution of bonds $(\alpha=0.857)$. (b) Resistivity vs. $T$, at magnetic fields $0(\bullet)$ and $7 \mathrm{~T}(\mathrm{O})$. (c) Field dependence of the spin-wave stiffness at $T=40 \mathrm{~K}$.

with $y$, with a conductivity exponent $t$ much larger than predicted by standard percolatione. (b) Far more challenging, $\rho_{d c}$ of $\mathrm{Sm}_{0.55} \mathrm{Sr}_{0.45} \mathrm{MnO}_{3}$ changes from insulator to metal, in a first-order transition $\mathbf{b}$. Although most Mn oxides have continuous MITs, their $\rho_{d c}$ vs. $T$ curves often show rapid changes and hysteresis loops, in the same regime where cluster percolation seems to occur. (c) Varying magnetic fields, $\mathrm{Pr}_{0.7} \mathrm{Ca}_{0.3} \mathrm{MnO}_{3}$ also presents a first-order-like MITt. These abrupt transitions cannot be rationalized within standard percolation theory 8 , but they are found in regimes that exhibit some aspects of percolationt.

The theory recently presented by Burgy et al 1 relies on a CMR state above $T_{\mathrm{C}}$ made out of preformed $\mathrm{FM}$ 
clusters, with random magnetic moment orientation (a similar picture was also qualitatively discussed by Uehara et al.2). This inhomogeneous state is stable due to the presence of quenched disorder. The random orientation of the cluster magnetic moments can be achieved by having "walls" between them made out of the competing insulating phase. Otherwise, when two FM clusters with different orientations become in contact they will align their moments. An insulating wall can prevent this process, by drastically reducing the interaction between domains. However, when a relatively small magnetic field is switched on, these preformed large FM moments can easily rotate into the direction of that field. When this occurs the insulating walls are no longer needed to prevent moment alignment, and they melt. This last process leads to a percolation, namely, as the FM clusters rotate their moments they also slightly increase their size to occupy the space left by the insulating walls, allowing for charge conduction to occurb. Although this last step appears percolative it is likely non-standard and abrupt, since it involves large rounded clusters that are not very ramified (contrary to standard percolation), due to surface tension effects which are of relevance in a regime of phase competition.

It is the main purpose of the present paper to describe, phenomenologically, this percolation of smooth objects, believed to describe CMR manganites. It will be argued that first-order characteristics can be found in percolative processes where surface tension effects are relevant. It is important to clarify that the full description of the CMR process is far more involved, with the random distribution and subsequent field-induced alignment of FM moments playing a key role The first steps in that direction were already described 5 . Here our goal is the following: if the FM clusters are assumed already aligned (and in that case the spin degree of freedom can be dropped), how does the subsequent percolation occurs? It will be argued that this transition can be fairly abrupt, even discontinuous. The paper is organized as follows. In Section II a variety of non-standard forms of percolation are described, searching for situations where first-order transitions are induced. The main conclusion of this section will be that achieving a discontinuous behavior is highly nontrivial and difficult. In Section III, a novel solution is proposed involving deterministic rules within a cellular automaton system. A discussion of results is presented in Section IV, while Section V contains the main conclusions.

\section{FORMS OF PERCOLATION}

Standard percolation (SP) is a successful statistical model for a variety of heterogeneous systems 3 . It has been used to describe diffusion through porous media, conduction through random networks, and forest fires, among others. In spite of its successes, however, SP has several shortcomings, including the failure to account for correlation effects. Indeed, in SP each site (or bond) is occupied independently of its neighbors. This feature is responsible for the ramified structure of the usual percolation clusters. In Mn oxides the coexistence of large clusters has been reported 3 , but the cluster distribution -while complex and involving many lengths scales- is not of the very ramified form characteristic of SP. A possible explanation lies in the long-range character of the elastic distortions induced by holes in Mn oxides 9 , as well as surface tension effects as described in the next section. These factors provide a natural tendency toward smooth surfaces of coexisting metal-insulator clusters. In addition, in physical systems with phase separation, there is a local tendency to homogenize the landscape, minimizing the interface tension.

While simulations of models considering the elastic effects through cooperative Jahn-Teller phonons are CPU time expensive, the cluster rounding effects appear evep in simpler Hamiltonians, such as the one-orbital modelt (the actual form of this Hamiltonian has been extensively discussed in previous literature, and it will not be reproduced here). Monte Carlo (MC) results in Fig.2(a,b) illustrate this phenomenon. In the simulations, the Heisenberg coupling $J_{\mathrm{AF}}$ between localized $t_{2 g}$ spins is selected such that some regions of the studied lattice are in a FM state if they were isolated, while others present some competing spin arrangement (antiferromagnetic (AF) or flux 1 ). However, when the FM and non-FM regions are in contact, there is a considerable influence from one another, and the system tends to homogenize its transport properties. For example, the ladder in Fig.2(a) would prefer to be in a perfectly AF spin state if isolated. However, by immersing the ladder into a ferromagnet, the spins cant appreciably, allowing for substantial charge hopping across the ladder. A thin AF region cannot survive the "pressure" of a FM environment, and it transforms into a fairly good conductor. Fig.2(b) shows similar evidence, now using competing FM and flux states. A finger-like region emerging from the lowerhalf, with coupling favoring the flux phase, also does not survive in its original form but spins rotate to allow for conduction 10 . Modeling percolative manganites with the metal/insulating character of a link independent of its neighbors can only be a crude approximation, and it may cause the present discrepancy between theory and experiment. If the clusters could be made more 'rounded' the transition would be more abrupt, and hopefully in better agreement with real manganites. The exponent $t$ may depend on the short-scale real-space metal-insulator arrangement, and changing the typical cluster shapes may alter $t$ as well.

The physics of manganese oxides, in particular the abrupt change in resistivity as a function of both field and temperature, is supposed to emerge from the realistic gne- and two-orbital models that are widely studied (supplemented by the addition of quenched disorder). However, simulations using realistic Hamiltonians for manganites -containing the rounding effect 


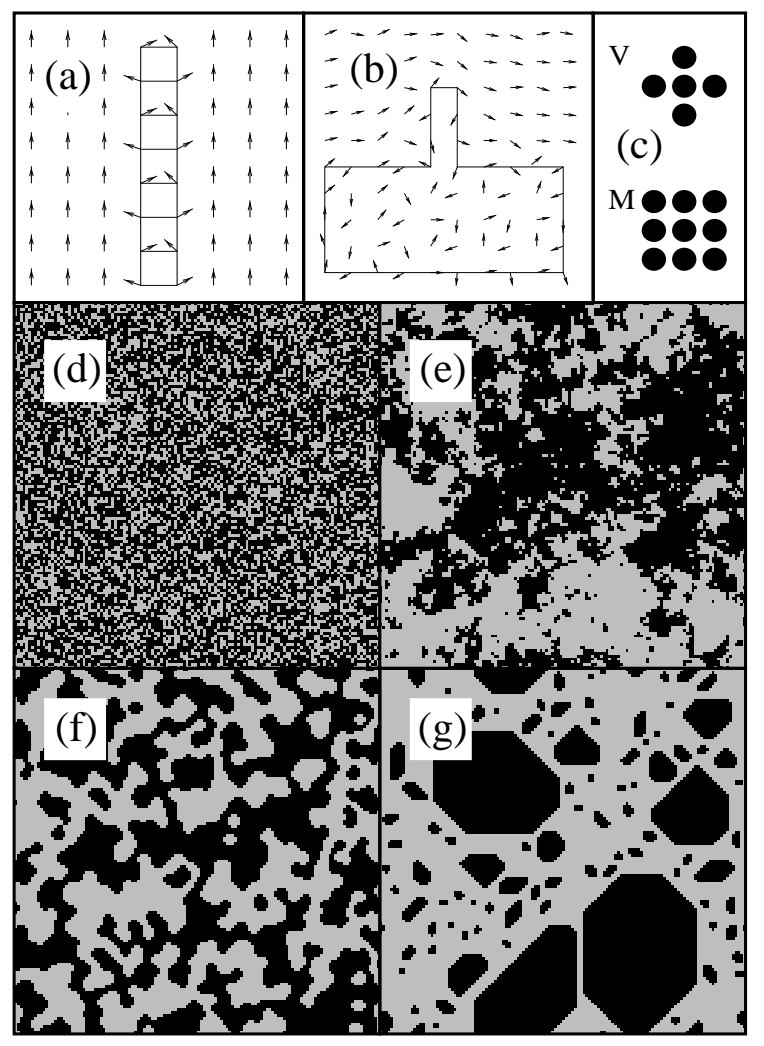

FIG. 2: (a) Typical snapshot of an $8 \times 8 \mathrm{MC}$ simulation of the one-orbital model (Hund coupling $J_{\mathrm{H}}=\infty, T=0.01 t, t$ the hopping amplitude, and density 0.8). $J_{\mathrm{AF}}$ is 0 everywhere, but in the bonds of the ladder where they take the value $0.12 t$. The average spin-spin correlation in the ladder is -0.4 (effective "double-exchange" hopping 0.54), as opposed to 1.0 (effective hopping 0) when isolated from the FM region (effective hopping 1). (b) Similar to (a) but for density 0.5. Here $J_{\mathrm{AF}}=0$ is 0 in the upper portion minus the finger-like region. In the rest $J_{\mathrm{AF}}=0.15 t$, favoring a flux state 4 . The effective hopping in the flux region is 0.7 , while in the finger it is as high 0.9. (c) The different neighborhoods in Vichniac's notation 16 . (d) Typical configuration for standard percolation on a $128^{2}$ lattice at (or just below) the critical density. (e) Same as (d) but for correlated disorder $(\gamma=0.2)$. (f) Same as (d) but for the annealing rule (MGE5). (g) Same as (d) but for the nucleating rule (MGE4). The latter is particularly promising since a small increase in $p$ leads to a fully saturated final state, in a first-order transition.

spontaneously- are simply prohibitive in the percolative regime if critical exponents are needed, and one must rely on semi-phenomenological approaches, as in the present paper. Even if we accept this simplification, there is, $a$ priori, no obvious way to introduce the proposed local cluster compactification in percolative scenarios. In alternatives to standard percolation such as "bootstrap" percolation 11 , one simply removes metallic sites or links if they do not have enough metallic neighbors. This is repeated until no more sites can be removed. However, numerical evaluation of the cluster number exponent $\tau$ suggests no new universal behavior 12 . To generate com- pact configurations, long-range "correlated disorder" was also investigated here 14 . The disorder is selected to follow the real-space correlation function $C(\ell)=\left(1+\ell^{2}\right)^{-\gamma / 2}$, which falls off like $\ell^{-\gamma}$ for large distances $\ell$ but is wellbehaved at $\ell=0$. Typical configurations confirm the presence of large clusters (Fig.2(e)). In addition, our conductivity studies for $\gamma=0.2$ (Fig.3) are compatible with a new universality class and $t>1.31=t_{\mathrm{SP}}$, as predicted before 15 . However, to reproduce the large exponent $t \sim 7$ found in experiments 6 anomalously small values of $\gamma$ are needed. The reason is that even with correlated disorder the weakest links still govern the critical behavior (Fig.2(e) shows weak links separating large clusters). Correlated disorder, with its statistical nature that allows for those links, is not sufficient to induce the smooth clusters needed to describe manganites. Naively, these already known results appear to rule out the possibility of finding an abrupt percolative transition to mimic the behavior of manganites.

Another alternative is the Swiss-cheese model17. Halperin et al.17 have shown that this model is equivalent to a random resistor network with a broad distribution of conductances $g$ given by

$$
P(g)=(1-p) \delta(g)+p(1-\alpha) g^{-\alpha} .
$$

Here $P$ denotes the probability that a conductance has values between $g$ and $g+\delta g, p$ is the metallic fraction as before, and $P(g)$ is characterized by an additional parameter $\alpha$. The weakest bond dominates in the effective resistance of the backbone. Extensive numerical tests 18 support the conjecture that $t=1 /(1-\alpha)$, for $0.25 \leq \alpha<1$. The previous equation becomes an equality for $\alpha$ large enough. Hence, since the data in Fig.1(b) follows a straight line with slope -7 , it can be fitted with results of a simulation at $\alpha \approx 0.857$. Although this numerical success is encouraging, it is still intuitively unsatisfactory and does not address the first-order percolative transitions of Figs.1(b,c). In fact, the rapid change in $\rho_{d c}$ in Fig.1(a) may also be indicative of a first-order transition hidden by imperfections in the samples used.

\section{FIRST-ORDER PERCOLATION}

The above examples clearly show that it is highly nontrivial to generalize percolation to yield a first-order transition, although it is possible to change the critical exponents. The existence of experiments (Figs.1(b,c)) with discontinuous transitions indicate that there must exist yet another class of percolative transitions escaping our attention. We believe that the key issue missing in standard percolation and the related variations described above is a mimic of the effect of surface tension, which naturally arises when two phases compete. This naturally penalizes the roughness of a surface and avoids the weak links ubiquitous in continuous percolation.

The simplest way to model the gregarious tendency of the two competing phases of manganites is to consider 


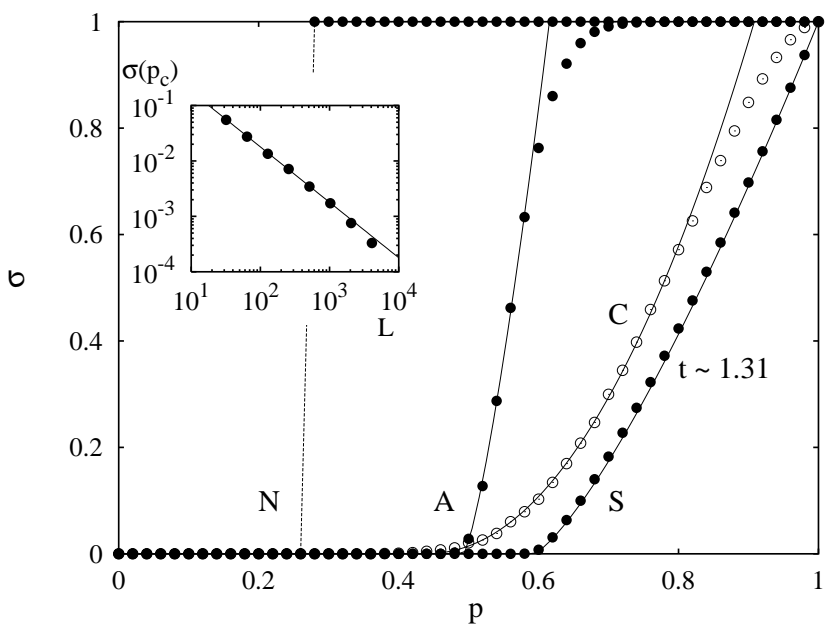

FIG. 3: Conductivity of a $2 \mathrm{D}$ resistor network vs. initial metallic fraction, for several representative cases. N, A, C, and S stand for nucleated (MGE4), annealed (MGE5), correlated, and standard, respectively. Both the $\mathrm{A}$ and $\mathrm{S}$ lines are proportional to $\left(p-p_{c}\right)^{t}$, with $t \sim 1.31$. The inset shows finite-size scaling data for A to support universality. $L$ is the linear size. Line $\mathrm{C}$ has a correlation power $\gamma=0.2$ and exponent $t \sim 2.1$.

the nature of the cells in the neighborhood of the cell under inspection. If there are enough "friendly faces" around, the cell will not change, otherwise it will flip to the competing phase. In a magnetic system, the neighborhood induces a local field that influences the center spin. In addition, including oxygen movement, it is impossible to have an undistorted octahedron in the middle of a distorted JT region. The phenomenological approach followed here is based on Vichniac's majority voting cellular automata16. Loosely speaking, cellular automata are discrete dynamical systems whose evolution is described by a local deterministic rule: the fate of every cell at time $t+1$ is determined by a function of the configuration of a few of its neighbors at time $t$. Cellular automata describe an approach to equilibrium in a system dominated by strong, short-range interactions. Vichniac 16 defines four different neighborhoods: Q contains only the nearest neighbors, V consists of $\mathrm{Q}$ plus the center cell, $\mathrm{H}$ consists of $\mathrm{Q}$ plus the second-nearest neighbors, and $\mathrm{M}$ consists of $\mathrm{H}$ plus the center cell (see Fig.2(c)). Unlike in bootstrap percolation 11, the center cell is treated on the same footing as its neighbors in $\mathrm{V}$ and $\mathrm{M}$. This turns out to be a key property of the percolative process discussed here, making clusters compact and avoiding weak links. This is quite different from bootstrap percolation where the center cell is singled out. After the neighborhoods are defined, the update rules are as follows. Given a site $i$ at a time $t$, let $S$ be the number of occupied sites in the neighborhood $N$ of $i . S$ can be any number between 0 and the number of sites in the neighborhood. If $S$ is greater than or equal to some threshold $T$, site $i$ will be occupied at time $t+1$. Otherwise it will be eppty. The procedure stops when no more changes occur 19 . A rule is specified by a unique $N$ and $T$. This is referred to as NGET 16 . For the four neighborhoods defined above, only 8 rules (with $T$ close to half of the number of sites in $N$ ) are nontrivial, as explained below.

The starting point for the calculations are always standard percolation configurations, characterized by an occupation probability $p$. At time $t=0, p$ is a good measure of the occupied fraction, within statistical fluctuations. Under the "time evolution" provided by the cellular automaton, the occupied fraction needs no longer be equal to $p$. Rules with large (small) $T$ tend to (increase) decrease it. For initial states with $p<p_{c}$, the final state does not percolate. Two examples, to be discussed in more detail in the next paragraph, are showed in Figs.2(f,g). Note the clear visual difference from the more canonical cases Figs.2(d,e). The clusters are now more rounded, and our intuitive goal of phenomenologically mimicking the effect of surface tension has been reached. After the time evolution stops, the resistivity is measured using a numerically exact algorithm 20 .

The increase in conductivity $\sigma$ immediately after $p_{c}$ $(=0.5)$ is clearly faster in the annealed (MGE5, i.e. each site remains or becomes metallic if five or more of its nine neighbors -including itself- are metallic) case than in standard percolation, as Fig. 3 indicates. This is in agreement with our intuition that rounding effects would speed up percolation, mimicking better the experimental situation after the randomly oriented FM clusters align in a magnetic field. However, it is surprising that this does not affect the value of $t$, as the inset shows. Nevertheless, this rapid increase could be experimentally relevant. On the other hand, the nucleating rule (MGE4, i.e. each site remains or becomes metallic if four or more of its nine neighbors -including itself- are metallic) leads to different results. Its resistivity jumps from 0 below $p_{c}$, where there is no connection across the system (see Fig.2(g)), to the fully saturated value above $p_{c}$. To understand this, note that the clusters in Fig.2(g) have very clean boundaries. Adding a single metallic site immediately outside one of the clusters will flip all the sites touched by the boundary. At $p$ close to $p_{c}$, it is likely that this apparently small perturbation will cause the cluster to coalesce with a neighboring cluster. In practice, this starts a chain reaction (avalanche) that only stops when the entire lattice turns metallic. The nucleating procedure indeed provides a percolative transition that is first-order.

How common are the first-order characteristics expected to be in the cellular automata models described here? Using the four $(\mathrm{Q}, \mathrm{V}, \mathrm{H}, \mathrm{M})$ neighborhoods defined above on the square lattice, there are eight non-trivial rules (QGE2, QGE3, VGE3, HGE4, HGE5, MGE4, MGE5, MGE6). By non-trivial we mean rules whose transition occur at a finite value of $p$ (i.e. neither at 0 nor at 1 ) in the infinite system limit. In all cases a careful size extrapolation was carried out numerically to determine the critical concentration. They are respectively $(0.16$, 
$0.83,0.5,0.33,0.66,0.25,0.5,0.75)$. Although these values are close to the fractions $(1 / 6,5 / 6,1 / 2,1 / 3,2 / 3$, $1 / 4,1 / 2,3 / 4)$, we know of no rigorous result that they are equal to them. In two cases not listed above (VGE2 and VGE4) the numerical uncertainty prevented us from determining for sure whether the rule is trivial or not. To be specific, the critical $p$ for VGE2 is very small, and it becomes smaller still as we increase the size of the system used in the simulation. We could not rule out the fact that $p$ may extrapolate to zero in this case. As a consequence, we chose to leave those two cases out of the present discussion. Of the eight rules, the conductivity of three of them (QGE2, HGE4 and MGE4) was found to vary discontinuously at the transition. The conductivity of MGE4 is shown in Fig. 3 where the reader can convince oneself that the transition is discontinuous. QGE2 and HGE4 look identical apart for a shift in the location of $p_{c}$. Phenomenologically, the three cases behave similarly in the sense that states very close to the transition are unstable against flipping even just one site. Hence, we conclude that first-order tendencies are certainly not a fragile feature among the set of possible voting cellular automata. However, far more work is needed to fully understand the behavior of these novel systems, with the present paper hopefully providing the first steps in that direction. The detailed analysis of the models introduced here may be as rewarding as the many previous studies of standard percolation.

\section{DISCUSSION}

The novel discontinuous percolative transition described here can be applied phenomenologically to the Mn oxides. In a previous related effort, Mayr et al.21 constructed random resistor networks using standard percolation and provided a tentative phenomenological description of manganites. The curves $\rho_{d c}$ vs. $T$ mimicked properly the results corresponding to some manganites, but were too smooth to accommodate the materials of Fig.1 as special cases. The nucleating-rule procedure to generate metal and insulator clusters described here may solve this problem. The left panel of Fig. 4 contains the effective resistivity (obtained solving the Kirchoff equations) vs. $T$ for configurations generated by the nucleating-rule, where the light (dark) regions in Fig.2(g) are insulating (metallic). The individual resistivity of those regions is taken from experiments, and they carry a $T$ dependence, similarly as in Mayr et al21. The new curves are in excellent agreement with experiments (see, e.g., Fig.1(b)), including the discontinuity, and the fact that there is a common value of $\rho_{d c}$ at both low- and high- $T$.

Note that the new family of percolative processes presented here naively seems triggered only by the occupation fraction $p$, not by temperature, as opposed to some of the results of Fig. 1. However, the ferromagnetic fraction in real manganites is a function of temperature: clusters
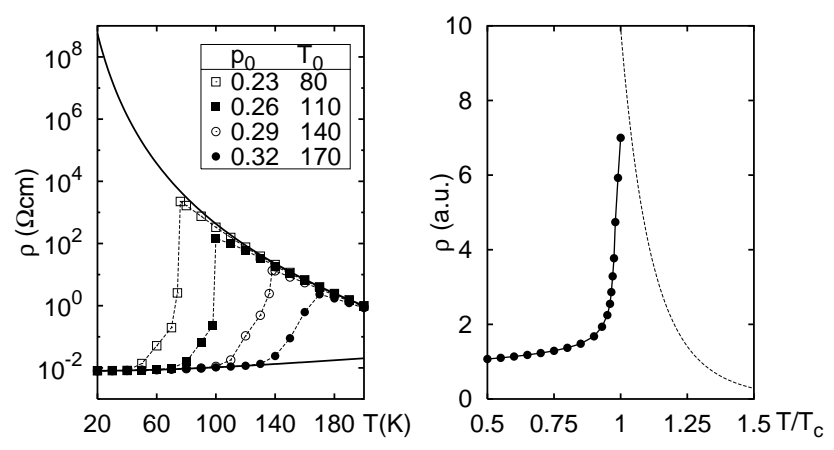

FIG. 4: Left panel: Resistivity $\rho_{d c}$ vs. $T$ using the nucleating rule. The values of $\rho_{d c}$ for metallic and insulating bonds are taken from experiments 3 , as in 21 . Following 21 , the metallic fraction varies linearly with temperature, $p-p_{0}=\alpha\left(T_{0}-T\right)$. We consider $\alpha=1 \%, T_{0}=100 \mathrm{~K}$, and label the curves by $p_{0}$, their metallic fraction at $T=T_{0}$. Right panel: $\rho_{d c}$ vs. $T$ of the $Q=20$ Potts model (for details, see text). A cluster algorithme2 was used on a $64^{2}$ lattice. Long simulations (several thousand sweeps) were required to sample both peaks in the probability distribution function satisfactorily. Above $T_{c}$ there is no percolation $\left(\rho_{d c}=\infty\right)$, but this can be solved phenomenologically by allowing for conductivity among the several $Q$ clusters, as in the dashed line.

are expected to be formed at $T^{*}$ (although they are not sufficiently large in size to create a global FM state), their number grows with decreasing temperature (i.e. $p$ increases in the language of this paper), until the Curie temperature is reached. It would be incorrect to believe that our results are only valid for cases where the temperature is held fixed while concentrations are changed.

Another clarification is important. Why do all manganites not have either a first- or second-order transition? Current experimental evidence suggests that both cases can be found, although the latter tends to be fairly abrupt 4 . Several aspects may be influencing these results. First, it may occur that available single crystals are affected by extrinsic effects in their preparation. From this perspective, the current experimental results may vary in the future as cleaner samples are prepared. But there are other issues to consider. Clearly, different manganites have different degrees of intrinsic disorder, such as the chemical disorder emerging from having different ionic sizes at the A-site of the perovskite. This disorder will likely influence the clusters shape, and both configurations Fig.2(f) and 2(g) may emerge at different compositions, leading to either rapid but continuous or firstorder percolative processes, respectively. It is clear that the issue of universality of the first-order transition in manganites is far from clear both experimentally and theoretically and more work should be devoted to its analysis. Here, we have simply provided a plausible rationalization of some steps involved in the CMR transition of the materials mentioned in Fig. 1. Whether all CMR materials have the same kind of first-order transitions or not will hopefully be clarified by future experiments. 
Finally, although the discussion here is based on a cellular automaton, we found it reassuring that first-order percolative processes can also arise from well-defined Hamiltonians. In particular, consider the $Q$-states Potts model known to have a a first-order transition for $Q>423$. Simulating the model $(Q=20)$ with $\mathrm{MC}$ techniques and using the Fortuin-Kasteleyn mapping 13 to construct clusters for the various $Q$ 's, their percolative properties were analyzed24. Clusters for each $Q$ are individually assumed metallic, and the network resistance is calculated. Results (Fig.4 (right)) indeed have a $\rho_{d c}$ discontinuity (above $\rho_{d c} \approx 7$ ) at the critical temperature $T_{c}$. At $T>T_{c}$, no cluster percolates leading to infinite $\rho_{d c}$, but this can be easily fixed assuming a finite conductivity among the many $Q$ clusters. Of course, it is not at all claimed here that real manganites have Potts symmetry, this is just an illustration of concrete model Hamiltonians that presents the first-order transition described in the previous section by cellular automata rules (rather than emerging from a Hamiltonian approach).

\section{CONCLUSIONS}

In summary, a phenomenological approach is proposed to include correlation and surface tension effects in perco- lation. The rounding of clusters induces an abrupt firstorder transition in the model, consistent with results of some experiments Fig.1(b). The present calculation suggests that many manganites do not belong to the standard percolation class, as believed by many before, but correlation effects change the universality class inducing a faster percolative process that may present hysteretic features25. Note that the fairly "random looking" configuration Fig. $2(\mathrm{~g})$ is self-generated 26 by our approach, its phenomenological origin lies in phase competition, and is not pinned by explicit disorder. Thus, the first-order percolative transition is predicted to appear particularly in nominally "clean samples" and for low intrinsic chemical disorder. If explicit disorder is added to the system Fig.2 $(\mathrm{g})$, then the transition is expected to become continuous. More work is needed to fully clarify the fascinating physics of CMR manganites.

This work was supported in part by NSF grant DMR0122523, and also by MARTECH (FSU).
1 A. Moreo, S. Yunoki and E. Dagotto, Science 283, 2034 (1999).

2 M. Uehara, S. Mori, C. H. Chen and S.-W. Cheong, Nature 399, 560 (1999).

3 M. Fäth, S. Freisem, A. A. Menovsky, Y. Tomioka, J. Aarts and J. A. Mydosh, Science 285, 1540 (1999).

4 E. Dagotto, T. Hotta and A. Moreo, Phys. Rep. 344, 1 (2001). See also E. Dagotto, Nanoscale Phase Separation and Colossal Magnetoresistance, Springer-Verlag, October 2002.

5 J. Burgy, M. Mayr, V. Martin-Mayor, A. Moreo and E. Dagotto, Phys. Rev. Lett. 87, 277202 (2001).

${ }^{6}$ E. Saitoh, Y. Tomioka, T. Kimura and Y. Tokura J. Phys. Soc. Jpn. 69, 2403 (2000).

7 J. A. Fernandez-Baca, P. Dai, H. Kawano-Furukawa, H. Yoshizawa, E. W. Plummer, S. Katano, Y. Tomioka, and Y. Tokura, Phys. Rev. B 66, 054434 (2002)

8 Introduction to Percolation Theory, by D. Stauffer and A. Aharony, Taylor \& Francis publishers, 1994.

9 D. I. Khomskii and K. I. Kugel, cond-mat/0112340.

10 The analog phenomenon -thin FM conductors turning insulator in an insulating background- also occurs.

11 J. Chalupa, P. L. Leath and G. R. Reich, J. Phys. C 12, L31 (1979).

12 C. M. Chaves and B. Koiller, Physica A 218, 271 (1995). The Ising model is also relat to percolation through the Fortuin-Kasteleyn mapping 13 . See also P. J. Bastiaansen and H. J. F. Knops, J. Phys. A 30, 1791 (1997); D. A. Wollman, M. A. Dubson and Q. Zhu, Phys. Rev. B 48, 3713 (1993).
13 C. M. Fortuin and P. W. Kasteleyn, Physica 57, 536 (1972).

14 H. A. Makse, S. Havlin, M. Schwartz and H. E. Stanley, Phys. Rev. E 53, 5445 (1996).

15 A. Weinrib, Phys. Rev. B 29, 387 (1984) predicted that correlations are relevant if $\gamma \nu<2$, where $\nu$ is the correlation-length exponent for standard percolation.

16 G. Y. Vichniac, Physica D 10, 96 (1984).

17 B. I. Halperin, S. Feng and P. N. Sen, Phys. Rev. Lett. 54, 2391 (1985); S. Feng, B. I. Halperin and P. N. Sen, Phys. Rev. B 35, 197 (1987).

18 M. Octavio and C. J. Lobb, Phys. Rev. B 43, 8233 (1991).

19 However, some configurations oscillate with period 2.

20 D. J. Frank and C. J. Lobb, Phys. Rev. B 37, 302 (1988).

21 M. Mayr, A. Moreo, J. A. Vergés, J. Arispe, A. Feiguin and E. Dagotto, Phys. Rev. Lett. 86, 135 (2001).

${ }^{22}$ R. H. Swendsen and J. S. Wang, Phys. Rev. Lett.58, 86 (1987); J. S. Wang and R. H. Swendsen, Physica A 167, 565 (1990).

23 R. J. Baxter, J. Phys. C, 6, L445 (1973).

24 F. Peruggi, Physica A 141, 140 (1987); S. Fortunato and H. Satz, Nucl. Phys. B 623, 493 (2002).

25 The complex dynamical behavior of manganites observed experimentally (see, e.g., I. G. Deac, S. V. Diaz, B. G. Kim, S.-W. Cheong, and P. Schiffer Phys. Rev. B 65, 174426 (2002)) could have its origin in the unusual percolative process described here.

26 J. Schmalian and P. G. Wolynes, Phys. Rev. Lett. 85, 836 (2000). 